\title{
Multiphoton Graph States from a Solid-State Single-Photon Source
}

Jin-Peng Li, ${ }^{\dagger, \ddagger}, \perp$ Jian Qin, ${ }^{\dagger, \ddagger, \perp}$ Ang Chen, ${ }^{\dagger, \ddagger}$ Zhao-Chen Duan, ${ }^{\dagger, \ddagger}$ Ying Yu, ${ }^{*}, \boldsymbol{\Phi}$

YongHeng Huo, ${ }^{,+\dagger, \ddagger}$ Sven Höfling, ${ }^{\ddagger}, \xi_{,} \|$Chao-Yang Lu, ${ }^{\dagger, \ddagger}$ Kai Chen, ${ }^{*, \dagger}, \ddagger$ and Jian-Wei Pan ${ }^{\dagger, \ddagger}$

$\dagger$ †efei National Laboratory for Physical Sciences at Microscale and Department of Modern Physics, University of Science and Technology of China, Hefei, Anhui 230026, China $\ddagger$ Shanghai Branch, CAS Center for Excellence and Synergetic Innovation Center in Quantum Information and Quantum Physics, University of Science and Technology of China, Shanghai 201315, China

IState Key Laboratory of Optoelectronic Materials and Technologies, School of Electronics and Information Technology, School of Physics, Sun Yat-sen University, Guangzhou, Guangdong 510275, China

$\S$ Technische Physik, Physikalisches Institut and Wilhelm Conrad Röntgen-Center for Complex Material Systems, Universität Würzburg, Am Hubland, D-97074 Würzburg, Germany

\|SUPA, School of Physics and Astronomy, University of St. Andrews, St. Andrews KY16 9SS, United Kingdom

$\perp$ These authors contributed equally to this work

E-mail: yuying26@mail.sysu.edu.cn; yongheng@ustc.edu.cn; kaichen@ustc.edu.cn 


\begin{abstract}
Photonic graph states are underlying resources for one-way optical quantum computation, quantum error correction, fundamental test of quantum mechanics, and quantum communication network. Most of existing works, however, are based on spontaneous parametric down-conversion source that intrinsically suffers from probabilistic generation and double pair components. Here, we create two important classes of graph states, a polarization-encoded four-photon Greenberger-Horne-Zeilinger (GHZ) state and a linear cluster state, by actively demultiplexing a deterministic single photon source from a semiconductor quantum dot embedded in a micropillar. A state fidelity of $0.763 \pm 0.004(0.790 \pm 0.009)$ and a count rate of $\sim 13 \mathrm{~Hz}$ are observed for the four-photon cluster (GHZ) state. The results constitute a new route towards the multiphoton entanglement with deterministic single-photon sources.
\end{abstract}

\title{
Keywords
}

Multiphoton entanglement graph states, four-photon GHZ state, four-photon linear cluster state, one-way quantum computation, solid-state single-photon source, semiconductor quantum dot 
Photons have been an important workhorse for quantum information technology, such as optical quantum computing, ${ }^{1}$ quantum simulation, ${ }^{2}$ quantum enhanced measurement, ${ }^{3}$ and quantum communication network. ${ }^{4}$ Almost all these processes involve a central source of multiphoton entanglement. ${ }^{5}$ As an important type of multiphoton entangled state, optical graph states, ${ }^{6,7}$ such as Greenberger-Horne-Zeilinger (GHZ) states ${ }^{8}$ and cluster states, ${ }^{7,9}$ is key resource of one-way optical quantum computation, ${ }^{9,10}$ quantum error correction, ${ }^{11}$ quantum communication network, ${ }^{12}$ and fundamental test of quantum mechanics. ${ }^{13}$

It has been 20 years since the proposal of one-way quantum computation based on graph states. Many schemes have been proposed to produce large-scale optical graph states, ${ }^{14-16}$ together with significant progress to experimentally generate optical graph states. ${ }^{17-20} \mathrm{Un}$ fortunately, most of these experiments are relying on probabilistic generation of photon-pair sources, in which there are inevitable multiphoton pair events and only few percent generation probability. ${ }^{21-23}$ This will seriously degrade entanglement's quality and reduce number of entangling photons, particularly for optical cluster state,${ }^{20}$ which impose large limits on their applications in quantum information processing (QIP). Recently, near-optimal spontaneous parametric down-conversion (SPDC) photon-pair sources have been used to generate multiphoton entanglement states including entangled 12 photons ${ }^{22}$ and 18 qubits. ${ }^{23}$ Despite these impressive results, deterministic light sources are needed to further develop QIP applications based on photons in a push-button manner.

Solid-state quantum emitters as intrinsically deterministic quantum light sources, have attracted a great deal of attention. ${ }^{24}$ Particularly, semiconductor quantum dot (QD) has been demonstrated as a promising candidate for on-demand generating high-quality single photons, ${ }^{25}$ and two-photon entangled states. ${ }^{26,27}$ The QD was also proposed to deterministically generate large scale cluster states, ${ }^{28-30}$ which has already been demonstrated in a proof-ofconcept experiment between the QD spin and two photons in polarization, and all photons travel in a single spatial mode. ${ }^{31}$ However, the very low generation rates and the length of cluster string is limited to the spin coherence time, ${ }^{31}$ which becomes considerable challenges 
to allow small-scale applications and further scalability. Thanks to the rapid progress of the high performance single-photon source (SPS) in recent years, ${ }^{25}$ there has possibility to generating multiphoton entanglement states by demultiplexing single push-button SPS and entangling gates, and photons travel in multi-spatial modes. By this way, one can already do some small-scale application research, which means a beginning for doing something that can only be performed by SPDC sources before. Additional, with the continuous improvement of the comprehensive performance of SPS, this method can even expect to exceed the upper limit performance of the SPDC source in multiphoton experiments in the future.

In this work, we experimentally demonstrate generation of polarization-encoded and individually-spatial-addressable photonic graph states by actively demultiplexing a deterministic SPS and post-selection entangling gates. The SPS based on a single QD embedded in a micropillar has high efficiency, purity and indistinguishability simultaneously. We are able to generate high fidelity and high brightness of four-photon GHZ state and linear cluster state, with the help of low loss active optical switches, high efficiency interferometer as well as superconducting nanowire single-photon detector. ${ }^{32}$ We also discuss the potential of our system and compare its scalability with the best SPDC source nowadays. ${ }^{22}$

As shown in Fig. 1, a single self-assembled InAs/GaAs QD is embedded inside a 2- $\mu \mathrm{m}$ diameter randomly etched circular micro-pillar cavity (for more details on the sample growth and fabrication, please see the note 28 of Ref. ${ }^{33}$ ). To reach the best QD-microcavity coupling with optical resonance around $893 \mathrm{~nm}$, the whole sample wafer was placed inside a closed recycle cryostat and cooled to $\sim 4 \mathrm{~K}$. Under pulse resonant excitation with a repetition rate of $76 \mathrm{MHz},{ }^{34,35} \sim 6.4 \mathrm{MHz}$ polarized resonance fluorescence (RF) single photons are detected (with detector efficiency $\sim 0.8$ ), has lifetime $\sim 68$ ps. The measured second-order correlation function at zero-time delay is 0.03(1), which indicates intrinsic nature of the single-photon source. The indistinguishability is measured by a Hong-Ou-Mandel interferometer, with a visibility of 0.91(1) between two photons separated by $6.5 \mu \mathrm{s} .{ }^{33}$ Such a long stream of neartransform-limited single photons makes it feasible to implement multiphoton entanglement 


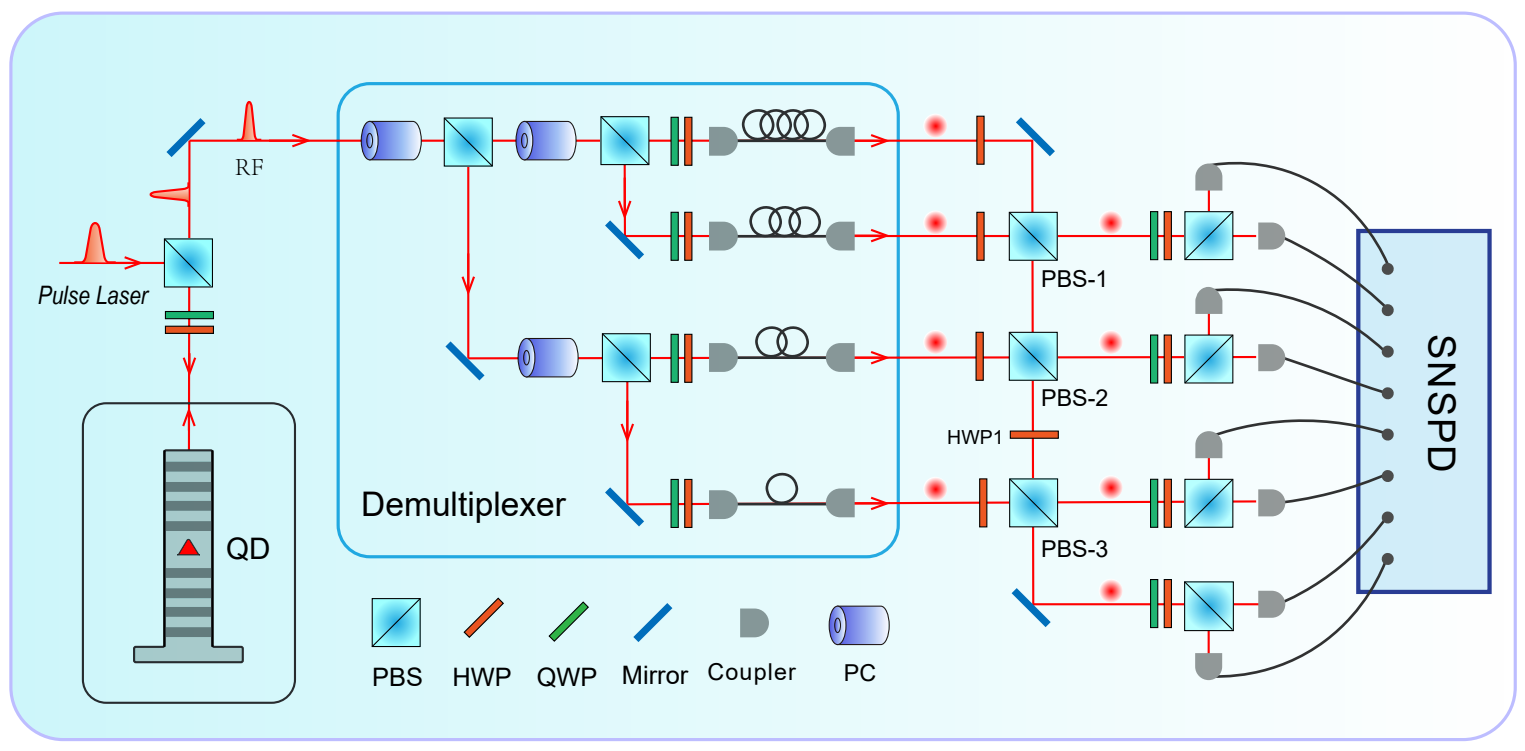

Figure 1: Experiment setup. The quantum dot (QD) sample is placed at a closed-cycle cryostat, and cooled to $\sim 4 \mathrm{~K}$. A pulse laser with a central wavelength around $893 \mathrm{~nm}$ excites the QD resonantly through fiber coupled confocal microscope system (without draw). A high extinction ratio $\left(>10^{5}: 1\right)$ polarizing beam splitter (PBS) and a pair of half- and quarter-wave plates (HWP, QWP) are used to extinguish the residual pump laser in cross polarization. Resonate fluorescence $(\mathrm{RF})$ single-photon stream is actively demultiplexed into four free spatial mode by three fast optical switches, each one constructed by a Pockels cell (PC) and a PBS. Four streams are then coupled respectively into four single-mode fibers. The different length of fibers and four translation stages (without draw) are used to finely compensate relative time delay of four photon streams. Time synchronized photons are prepared into polarization state $|+\rangle$ by wave plates, and then simultaneously fed into interferometer (PBS1-3). The HWP1 between PBS2 and PBS3 are used to switch generation between $\mathrm{GHZ}$ and cluster state by setting it at $0^{\circ}$ or $22.5^{\circ}$. A pair of wave plates and $\mathrm{PBS}$ at each output are used to do polarization analysis. Finally, the output photons are detected by fiber-coupled superconducting nanowire single-photon detector (SNSPD) and registered by a homemade coincidence count unit. ${ }^{32}$ The average efficiency of optical switches, optical lines and SNSPDs is about $0.83,0.80$ and 0.70 respectively

experiments.

Afterwards, the single-photon stream is deterministically demultiplexed into four free spatial modes by three cascade fast optical switches (see Fig. 1). ${ }^{32}$ Each switch consists of a polarizing beam splitter (PBS, with an extinction ratio $>2000: 1$ ) and an active high voltage Pockels cell (PC), which on-demand rotates the photon polarization by $90^{\circ}$ driven by $\sim 1800$ volts (with an extinction ratio $>100: 1$ ). The PCs are synchronized to the pulse laser 
and operated at a repetition rate of $0.76 \mathrm{MHz}$. In each period, every 25 sequential photon pulses are actively imported into one of four free spatial modes by optical switches, before collected into four single-mode fibers. Each fiber has different length to roughly compensate time delay among four modes, then four translation stages applied to make perfect temporal overlap (not draw in Fig. 1). Thanks to the high transmission rate of PC $(>99 \%)$ and high single-mode fiber coupling efficiency $(\sim 85 \%)$, the average efficiency of demultiplexers reaches about $83 \%$ for each photon stream mode. This type of multiple SPSs are generated from the same SPS, thus eliminating the photon distinguishability from different SPSs. ${ }^{36,37}$ This will ensure the high contrast of multiphoton interference. ${ }^{5,38}$ Moreover, the demultiplexer is deterministic and does not introduce extra loss in principle. Finally, each single photons can be manipulated in temporal and spatial mode flexibly, which is crucial for a large-scale QIP.

Time synchronous single photon streams are prepared into $|+\rangle=(|H\rangle+|V\rangle) / \sqrt{2}$ state by a pair of half- and quarter-wave plates (HWP and QWP), where $H(V)$ represents horizontal (vertical) polarization. Then photons are fed into three PBSs (PBS1-3), each of which acts as the core of post-selection entangling gate. As the PBS transmits $H$ and reflects $V$ polarization, only if both incoming photons have the same polarization can they go to different outputs. Thus, when HWP1 is set at $0^{\circ}$ (see Fig. 1), there is one-eighth probability for four-fold coincidence of all four output modes, which corresponds to a successful generation of the state:

$$
\left|G_{4}\right\rangle=\frac{1}{\sqrt{2}}\left(|H\rangle_{1}|H\rangle_{2}|H\rangle_{3}|H\rangle_{4}+|V\rangle_{1}|V\rangle_{2}|V\rangle_{3}|V\rangle_{4}\right)
$$

It is a four-photon polarization-entangled GHZ state, an equal superposition of two fully distinguishable quantum states. This is because, for ideal pure and indistinguishable four single photons interfering at the ideal PBS-based interferometer, there will be 16 photon polarization combinations which are equal probability coherent superposition. Each component corresponds to a special spatial modes and photon number distribution combination. Such as $|H\rangle_{1}|H\rangle_{2}|H\rangle_{3}|H\rangle_{4}$ or $|V\rangle_{1}|V\rangle_{2}|V\rangle_{3}|V\rangle_{4}$ corresponds to each one of four output spatial modes has only one photon. Different from other components, only they can contribute 
to the four-fold coincidence events and these two possibilities are quantum mechanically indistinguishable. Once detect a four-fold coincidence event, that means one have successfully picked out the GHZ state components by post-selection. We also note that, for this method of generating states, one can also do some quantum gates to accomplish quantum algorithm before pick out the target prepared states by coincidence-detection photons. Such as measurement based quantum computation ${ }^{10,18}$ and some shallow optical quantum circuit, ${ }^{5,34}$ can be realized equally to the method of deterministic generating states. The only limitation is that in the final photon detection process, we can still distinguish and pick out the wanted evolution process from other branches (for example, through other freedoms of degree), which is designed to operated on the target preparation state. In the similar way, one just inputs two or three photons, then two-photon Bell state ${ }^{39}$ or three-photon GHZ state can be generated conveniently. ${ }^{8}$

When the HWP1 is set at $22.5^{\circ}$, the above scheme can be readily modified to generate a four-photon polarization-entangled linear cluster state as follows:

$$
\begin{aligned}
\left|C_{4}\right\rangle= & \frac{1}{2}\left(|H\rangle_{1}|H\rangle_{2}|H\rangle_{3}|H\rangle_{4}+|H\rangle_{1}|H\rangle_{2}|V\rangle_{3}|V\rangle_{4}\right. \\
& \left.+|V\rangle_{1}|V\rangle_{2}|H\rangle_{3}|H\rangle_{4}-|V\rangle_{1}|V\rangle_{2}|V\rangle_{3}|V\rangle_{4}\right),
\end{aligned}
$$

which is successfully prepared when the four-fold coincidence events of all four output modes is detected with a one-eighth probability. It is a special class of multipartite graph state. To transform into the standard four-photon linear graph state, one only apply a local-unitary operation $U=H_{1} I_{2} I_{3} H_{4}$, where $H(I)$ represents Hadamard (Identity) operator. This state displays strong entanglement between any two photons, ${ }^{7}$ and enjoys improved robustness to loss compared to the GHZ state. ${ }^{40}$ It is worth noting that this configuration can be easily extended to generate any photon number linear cluster state by sequentially adding PBS and Hadamard gate (HWP set at $22.5^{\circ}$ ), and also to produce high-dimensional cluster state by proper configuration. ${ }^{1,17,18}$

To evaluate the quality of generated graph state, we measure the state fidelity that is 

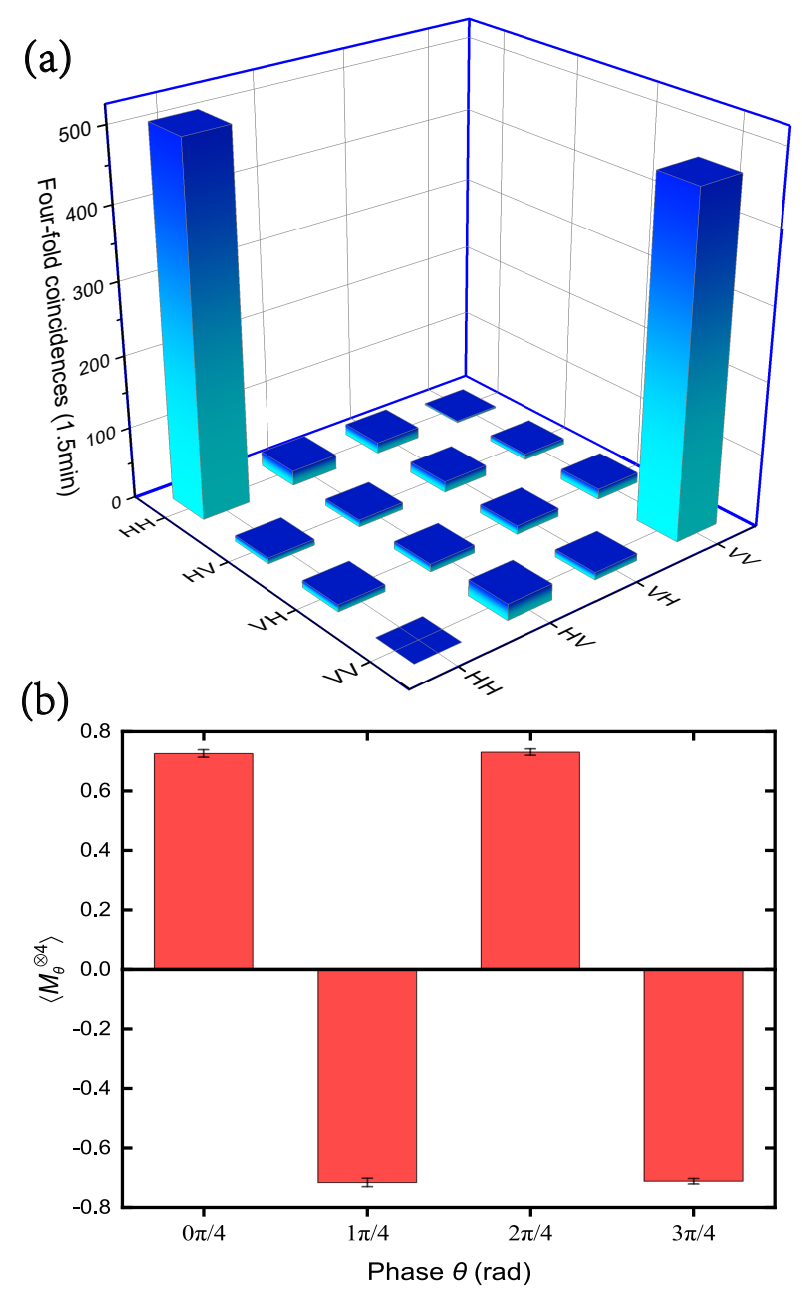

Figure 2: Experiment results of four-photon GHZ state. (a) Four-fold coincidence counts in $\mathrm{H} / \mathrm{V}$ basis with data collection in 1.5 minutes, the vaule of $\left\langle(|H\rangle\langle H|)^{\otimes 4}\right\rangle+\left\langle(|V\rangle\langle V|)^{\otimes 4}\right\rangle$ is $0.860(18)$. (b) The expectation values of $M_{k \pi / 4}^{\otimes 4}=\cos (k \pi / 4) \sigma_{x}+\sin (k \pi / 4) \sigma_{y} \quad(k=$ $0,1,2,3)$ obtained by the measurement in the basis of $\left(|H\rangle \pm e^{i k \pi / 4}|V\rangle\right) / \sqrt{2}$. Each basis accumulates about 1500 events in a few minutes. The error bar is derived from raw detection events by Poissonian counting statistics analysis with one standard deviation. The measured expectation values are 0.724(12), $-0.715(13), 0.729(10),-0.710(9)$ in order. 
overlap of experimentally generated state and the ideal one: ${ }^{41-43} F\left(\psi^{N}\right)=\left\langle\psi^{N}\left|\rho_{\operatorname{expe}}\right| \psi^{N}\right\rangle=$ $\operatorname{Tr}\left(\rho_{\text {ideal }} \rho_{\text {expe }}\right)$. Where $\left|\psi^{N}\right\rangle$ represents the ideal $N$-photon state, and $\rho_{\text {ideal }}\left(\rho_{\text {expe }}\right)$ denotes the density matrix of ideal state (experimentally generated state), the value of fidelity will be unity for a perfect match. For the GHZ state, $F\left(\psi^{N}\right)=\left(\left\langle P^{N}\right\rangle+\left\langle C^{N}\right\rangle\right) / 2$, with $\left\langle P^{N}\right\rangle=\left\langle(|H\rangle\langle H|)^{\otimes N}\right\rangle+\left\langle(|V\rangle\langle V|)^{\otimes N}\right\rangle$, which denotes the population of $|H\rangle^{\otimes N}$ and $|V\rangle^{\otimes N}$ components of the GHZ state. In addition,

$$
\left\langle C^{N}\right\rangle=\frac{1}{N} \sum_{k=0}^{N-1}(-1)^{k}\left\langle M_{k \pi / N}^{\otimes N}\right\rangle,
$$

with $M_{k \pi / N}=\sigma_{x} \cos (k \pi / N)+\sigma_{y} \sin (k \pi / N)$, where $\sigma_{x}$ and $\sigma_{y}$ are Pauli operators, $k=$ $0,1, \ldots, N-1$. The $\left\langle C^{N}\right\rangle$ is defined by the off-diagonal elements of the density matrix and reflects the coherent superposition between the $|H\rangle^{\otimes N}$ and $|V\rangle^{\otimes N}$ components of the GHZ state. The $M_{\theta}$ is a single qubit observable with eigenstate of $|\varphi\rangle^{ \pm \theta}=\left(|H\rangle \pm e^{i \theta}|V\rangle\right) / \sqrt{2}$ and eigenvalue of \pm 1 . The single qubit projective measurement can be realized by a pair of wave plates and a PBS (see Fig. 1).

The experimental data of the four-photon GHZ state are shown in Fig. 2. We observe $\sim 13 \mathrm{~Hz}$ four-fold coincidence count rate. Each measurement setting accumulates about 1,500 coincidence counts for computing the fidelity. Finally, we maintain a fidelity of $0.790 \pm 0.009$, which exceeds the entanglement threshold of 0.5 by 32.2 standard deviations. This ensures presence of genuine four-partitive entanglement, excluding possibilities from any partial entanglement (e.g.,biseparable states).

To evaluate the four-photon linear cluster state, we estimate its fidelity as $F_{C 4}=\left\langle C_{4}\left|\rho_{\text {expe }}\right| C_{4}\right\rangle$, by averaging 16 stabilizers' expectation values. ${ }^{42}$ As a four-photon linear cluster state, its stabilizer generators are:

$$
g_{1}=Z Z I I, g_{2}=X X Z I, g_{3}=I Z X X, g_{4}=I I Z Z
$$

where $X, Y, Z$, and $I$ represent Pauli operators $\sigma_{x}, \sigma_{y}, \sigma_{z}$, and Identity operator respectively. 

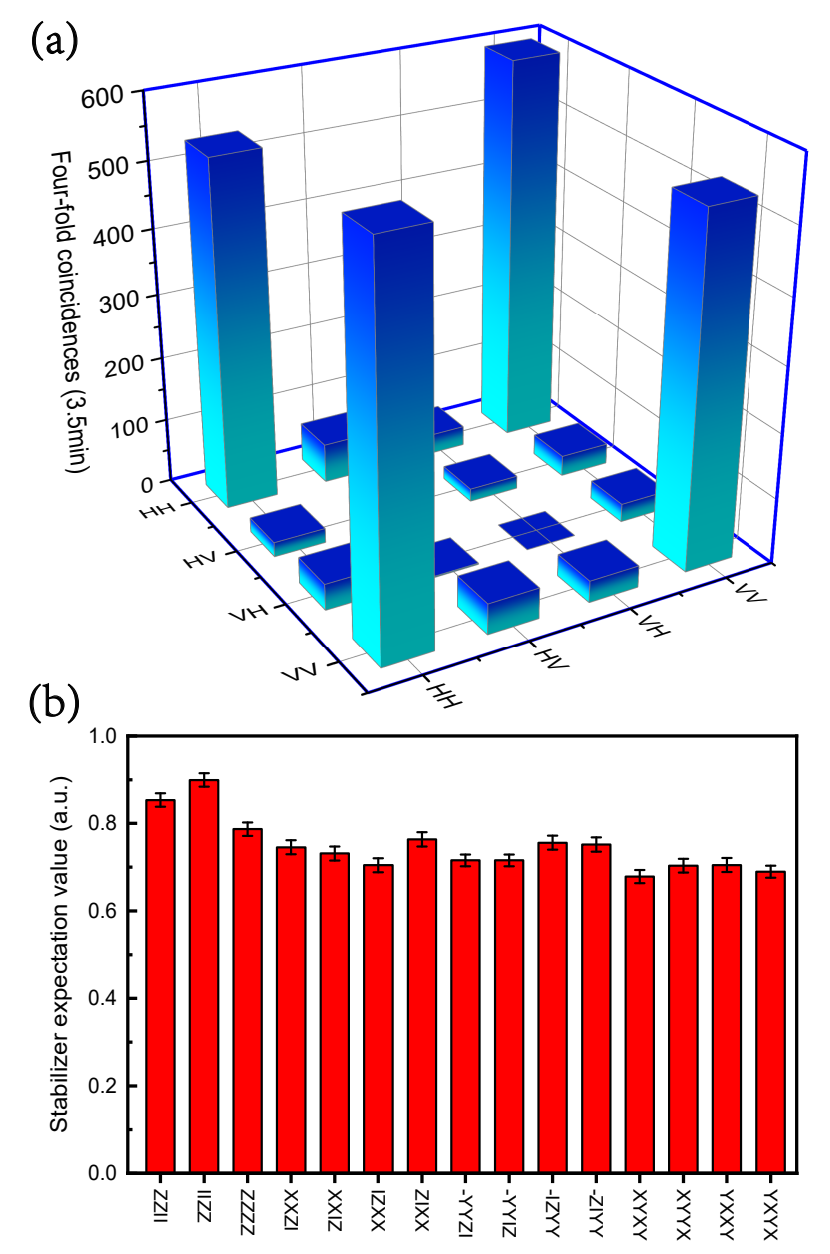

Figure 3: Experiment results of four-photon cluster state with QD works at the same excitation condition as generating GHZ state. (a) Four-fold coincidence counts acquired in $\mathrm{H} / \mathrm{V}$ basis. (b) The expectation values of stabilizers of the four-photon cluster state (the Identity stabilizer is not shown). Each setting has about one thousand coincidence counts accumulated in few minutes. The $I, X, Y, Z$ represent Identity matrix, $\sigma_{x}, \sigma_{y}, \sigma_{z}$ Pauli operators respectively. The error bar is deduced from propagated Poissonian counting statistics of the raw detection events with one standard deviation. The measured expectation values are $0.854(15), 0.899(15), 0.787(15), 0.745(15), 0.731(15), 0.704(16), 0.763(16), 0.716(14)$, $0.716(14), 0.756(16), 0.752(16), 0.679(15), 0.703(16), 0.705(16), 0.689(13)$ in order. 
All stabilizers can be derived from the stabilizer generators' cyclic products [as shown in the $x$-axis of Fig. 3(b)]. Each expectation value can be calculated from the probability of projecting into their eigenstates, whose all 16 combinations are recorded at the same time. As shown in Fig. 3, with the same excitation condition as generating GHZ state, we measure all the 16 stabilizers. Because of the non-ideal multiphoton interference, the expectation values of different stabilizers have fluctuations, but all greater than the classical threshold of 0.5. Finally, the fidelity of four-photon linear cluster state is shown to be $0.763 \pm 0.004$, which exceeds the threshold of 0.5 by 65.7 standard deviations, witnessing genuine multipartite entanglement with a large confidence. ${ }^{42}$

To verify non-locality of four-photon cluster state, the stabilizer correlations can also be used for constructing a Bell-type inequality with the following Bell operator: ${ }^{17}$

$$
S=Z I X X+X Y Y X+X Y X Y-Z I Y Y .
$$

The maximal expectation value of $S$ is $\operatorname{Tr}\left(S \rho_{c}\right)=4$ for the cluster state from quantum mechanics, while the bound for local hidden variable model is $S=2$. In our experiment we reach $2.897 \pm 0.032$, which clearly violates the classical bound 2 by 28 standard deviations, indicate high quality of the four-photon cluster state.

Compared to the early optical graph state experiments which was built on SPDC entangled photon pair (EPR) source, ${ }^{17,18,44}$ our generation state have better state fidelity and higher generation rate. This means that it is feasible to demonstrate one-way quantum computation in a way like Ref., ${ }^{18}$ and high fidelity quantum teleportation like Ref. ${ }^{44}$ by our generated states. However, the generated states are still imperfect, mainly due to non-unity purity and non-ideal indistinguishability of SPS. The non-unity purity of single photons means that there is a small fraction of multiphoton events in RF pulse, mainly comes from residual pump laser background and fluorescence of some other QDs. ${ }^{35}$ The non-unity indistinguishability will result in imperfect multiphoton interference. It is primarily caused 
by phonon sideband of RF and some residual effects leading to QD decoherence, ${ }^{25}$ such as charge and spin noise in environment around the QD. ${ }^{45}$ Both of them contribute to unwanted background of effective four-fold coincidence, which degrades the fidelity of generated state, and imposes an upper limit of the number of entangled photons. ${ }^{46}$ As a rough estimation, we use the fidelity of genuine two-photon Bell state $\sim 0.92$ (which is limited by the indistinguishability and purity of SPS), to estimate the fidelity of $N$-photon entanglement state $F\left(\psi^{N}\right)=0.92^{N-1}$, and when $N=9(10)$, the $F\left(\psi^{9(10)}\right)=0.513(0.472)$. Thus, the largest number of photons being entangled goes to nine. It is smaller than the optimal experiment results of 12 photons or 18 qubits based on SPDC source. ${ }^{22,23}$ However, in principle, the indistinguishability and purity of solid-state SPS can all be improved to near-unity. ${ }^{25,34}$ Thus, the fidelity of generated states by SPS can approach to one and the upper limit of entangled photons can be greatly extended. These strategies, such as low density and high quality QD wafer growth, better single QD-cavity coupling by optimizing cavity structure, ${ }^{47-49}$ and proper excitation method ${ }^{50-53}$ will help to approach an ideal SPS. ${ }^{25}$

As the number of entangled photons increase, a change in the production rate of the prepared state is another factor for performance, which is related to scalability of system. In fact, $N$-photons quantum experiments usually constitute three steps: preparing photons with probability $\eta_{\text {pre }}$ and repetition rate $R_{\text {rep }}$, operating photons states with successful probability $\eta_{\text {ope }}$ (such as entangled gate operations), and detecting photons with probability $\eta_{\text {det }}$. Then the probability of catching a $N$-fold coincidence event which evolve as we expected is $P_{\exp }=$ $\eta_{\text {pre }} \eta_{\text {ope }} \eta_{\text {det }}$, with $N$-fold count rate defined as $R_{C C N}=R_{\text {rep }} P_{\text {exp }}$. In our experiment (the best SPDC EPR source based experiment to date ${ }^{22}$ ), suppose the repetition of pump laser is $R_{L 1}$ $\left(R_{L 2}\right)$, and the average efficiencies for each photon are as following: photon brightness at fiber output end is $\eta_{f b 1}\left(\eta_{f b 2}\right)$, optical switches efficiency $\eta_{s 1}\left(\eta_{s 2}=1\right.$ they don't need optical switches), optical lines and interferometer efficiency $\eta_{l 1}\left(\eta_{l 2}\right)$, detecting efficiency $\eta_{d 1}\left(\eta_{d 2}\right)$, 
the $N$-fold coincidence count rate $R_{C C N 1}$ for our system is:

$$
R_{C C N 1}=\frac{R_{L 1}}{N} P_{e x p 1}=\frac{R_{L 1}}{N}\left(\left(\eta_{f b 1} \eta_{s 1}\right)^{N} \frac{1}{2^{N-1}}\left(\eta_{l 1} \eta_{d 1}\right)^{N}\right)
$$

where $\eta_{\text {ope } 1}=1 / 2^{N-1}$ denotes the successful probability of generation $N$-photon entanglement states (such GHZ and linear cluster states) by a minimal number of $N-1$ entanglement gates, which achieved by PBSs based interferometer and post-selection measurement. Bringing the actucal experiment parameters: $R_{L 1}=76 M H z, \eta_{f b 1} \approx 6.4 M / 0.8 / 76 M=0.105, \eta_{s 1} \approx$ $0.830, \eta_{l 1} \approx 0.80, \eta_{d 1} \approx 0.70$ into equation $(6)$, we compute $R_{C C N 1}=1.52 * 10^{8} * \frac{0.0244^{N}}{N}$, it will decrease exponentially with $N$ increases. However, it will be improved significantly by improving the efficiency of optical lines and detectors to near unity, and increasing the repetition rate of the pump laser at least 8 times.

For the best SPDC EPR source based experiment to date like Ref., ${ }^{22}$ the rate $R_{C C N 2}$ is:

$$
R_{C C N 2}=R_{L 2} P_{e x p 2}=R_{L 2}\left(\left(\eta_{f b 2}\right)^{N / 2} \frac{1}{2^{N / 2-1}}\left(\eta_{l 2} \eta_{d 2}\right)^{N}\right)
$$

For maximizing advantage of SPDC EPR source and easily deriving, here we suppose that $N$ is even. The EPR source always produce two entangled photons at the same time, so the $\eta_{p r e 2}=\left(\eta_{f b 2}\right)^{N / 2}$. We note that the $\eta_{f b 2}$ is approximately equal to the probability of generation single EPR. Because the EPRs is directly emitted from nonlinear crystals to free space, with the spacial mode almost ideal matching to single mode fiber in that experiment. ${ }^{22}$ The photons can be considered equivalent output from sing mode fibers. The item of $\eta_{\text {ope } 2}=\frac{1}{2^{N / 2-1}}$, denotes the successful probability of minimum $N / 2-1$ entangled gates for generating $N$-photons entanglement states (such GHZ and some cluster states) from $N / 2$ entangled photon pairs.

For facilitating comparison of the $R_{C C N}$ differences between them, and highlighting the main factors affecting scalability in production rate, we suppose $R_{L 1} / N=R_{L 2}, \eta_{l 1}=$ 
$\eta_{l 2}, \eta_{d 1}=\eta_{d 2}$ (the actual experimental parameters are almost like this), and define:

$$
K_{N}=R_{C C N 1} / R_{C C N 2}=\left(\frac{\eta_{f b 1} \eta_{s 1}}{\sqrt{\eta_{f b 2}}}\right)^{N} \frac{1}{2^{N / 2}}
$$

which denote the relative generation rate for generating the same $N$-photon entanglement states. With the real experiment parameters $\eta_{f b 1} \approx 6.4 M / 0.8 / 76 M=0.105, \eta_{s 1} \approx 0.830, \eta_{f b 2} \approx$ $0.050,{ }^{22}$ one has $K_{N}=0.333^{N}$. With $N$ increases, $K_{N}$ will decrease exponentially, denote the best SPDC source to date has a better scalability than our system. However, for SPDC EPR source of Ref., ${ }^{22} \eta_{f b 2}=0.050$ almost is its optimal value when consider the trade-off between high heralded efficiency and high single EPR productivity and purity. But, for our system, the $\eta_{f b 1}$ and $\eta_{s 1}$ can respectively gradually increase to one in principle. And, when $\eta_{f b 1} * \eta_{s 1}>\sqrt{0.05 * 2} \approx 0.316$ (nowadays the highest value of QD SPS based multi-SPSs is $\sim 0.217^{32}$ ), the $K_{N}$ will larger than one, means the scalability of SPS based configuration will be better than SPDC based. It is feasible that with the recent beautiful work reported methods by coupling QD to an asymmetric micro-cavity ${ }^{52}$ or pumping SPS by bi-chromatic laser pulse ${ }^{51}$ the fiber output brightness $\eta_{f b 1}$ of SPS will be hopefully reached to dramatically near unity. That will be a crucial step forward for helping implement scalable multi photon quantum experiments without SPDC EPR source.

In addition, based on recent progress by using natural or artificial atoms to engineer photon-photon interactions, ${ }^{54}$ improving the operation probability $\eta_{\text {ope }}$ to unity is also possible. Thus, the $R_{C C N 1}$ will almost not decrease when increasing entangled photon number $N$, the scalability will be ideal. Notably, another way to achieve high $\eta_{f b}$ SPS is to multiplex heralded SPSs which are from SPDC sources ${ }^{55}$ or four-wave mixing. ${ }^{56}$ Still, there are some challenges that limit their applications in multiphoton experiments, such as the trade-off between the purity of SPS and resource consumption, the lower repetition rate which limited by the speed of optical switches, and the switches losses increase with the multiplex modes in both spatial and temporal multiplexing. Therefore, to our knowledge, there has been no 
multiphoton entanglement experiment implement by this type SPS, although they already outperform existing QD sources on only certain individual indicator (refer to the discussion in Ref. ${ }^{55}$ ). For multiplex heralded SPS, it is still difficult to achieve high efficiency, high purity, high indistinguishability and high production rate at the same time, however, in principle it can also have the similar utility as QD based SPS.

In summary, we have for the first time generated both four-photon GHZ and cluster states in high fidelity and high count rate by a QD-micropillar based deterministic SPS, which is demultiplexed into four spatial modes by active fast photonic switches. The method can be readily extended to entangle more photons, and the scalability in production rate can be approach the best in principle, thus opening a new way for multiphoton entanglement experiments with a single deterministic SPS. Our results also led to a beginning, to do some experiments that could only be carried out with SPDC sources before, and even expect to exceed the upper limit performance of SPDC sources in the future. Many interesting experiments can be performed with this system in the near future, such as graph-state-based quantum error correction, ${ }^{57}$ triggered Bell-state generation, ${ }^{58}$ and demonstrate heralded universal quantum computation. ${ }^{1}$ Combined with high dimensional ${ }^{59}$ and multiple degrees of

freedom techniques, ${ }^{19,23}$ the system can also provide platforms for other promising quantum photonic experiments and applications. ${ }^{60}$

Note added.-During preparation of our manuscript, we note that there is one similar experiment for realization of four-photon cluster state. ${ }^{61}$

\section{Acknowledgement}

This work was supported by the National Natural Science Foundation of China, the Chinese Academy of Sciences, and the National Basic Research Program of China (973 Program). 


\section{References}

(1) Knill, E.; Laflamme, R.; Milburn, G. J. A scheme for efficient quantum computation with linear optics. Nature 2001, 409, 46-52.

(2) Aspuru-Guzik, A.; Walther, P. simulators. Nat. Phys. 2012, 8, 285-291.

(3) Giovannetti, V.; Lloyd, S.; Maccone, L. Advances in quantum metrology. Nat. Photonics 2011, 5, 222-229.

(4) Kimble, H. J. The quantum internet. Nature 2008, 453, 1023-1030.

(5) Pan, J.-W.; Chen, Z.-B.; Lu, C.-Y.; Weinfurter, H.; Zeilinger, A.; Żukowski, M. Multiphoton entanglement and interferometry. Rev. Mod. Phys. 2012, 84, 777.

(6) Hein, M.; Eisert, J.; Briegel, H. J. Multiparty entanglement in graph states. Phys. Rev. A: At., Mol., Opt. Phys. 2004, 69, 062311.

(7) Briegel, H. J.; Raussendorf, R. Persistent entanglement in arrays of interacting particles. Phys. Rev. Lett. 2001, 86, 910.

(8) Greenberger, D. M.; Horne, M. A.; Zeilinger, A. Bell's theorem, quantum theory and conceptions of the universe; Springer, 1989; pp 69-72.

(9) Raussendorf, R.; Briegel, H. J. A one-way quantum computer. Phys. Rev. Lett. 2001, 86,5188 .

(10) Briegel, H. J.; Browne, D. E.; Dür, W.; Raussendorf, R.; Van den Nest, M. Measurement-based quantum computation. Nat. Phys. 2009, 5, 19-26.

(11) Schlingemann, D.; Werner, R. F. Quantum error-correcting codes associated with graphs. Phys. Rev. A: At., Mol., Opt. Phys. 2001, 65, 012308.

(12) Markham, D.; Sanders, B. C. Graph states for quantum secret sharing. Phys. Rev. A: At., Mol., Opt. Phys. 2008, 78, 042309. 
(13) Gühne, O.; Tóth, G.; Hyllus, P.; Briegel, H. J. Bell inequalities for graph states. Phys. Rev. Lett. 2005, 95, 120405.

(14) Nielsen, M. A. Optical quantum computation using cluster states. Phys. Rev. Lett. 2004, 93, 040503.

(15) Browne, D. E.; Rudolph, T. Resource-efficient linear optical quantum computation. Phys. Rev. Lett. 2005, 95, 010501.

(16) Bodiya, T.; Duan, L.-M. Scalable generation of graph-state entanglement through realistic linear optics. Phys. Rev. Lett. 2006, 97, 143601.

(17) Kiesel, N.; Schmid, C.; Weber, U.; Tóth, G.; Gühne, O.; Ursin, R.; Weinfurter, H. Experimental analysis of a four-qubit photon cluster state. Phys. Rev. Lett. 2005, 95, 210502 .

(18) Walther, P.; Resch, K. J.; Rudolph, T.; Schenck, E.; Weinfurter, H.; Vedral, V.; Aspelmeyer, M.; Zeilinger, A. Experimental one-way quantum computing. Nature 2005, $434,169-176$.

(19) Chen, K.; Li, C. M.; Zhang, Q.; Chen, Y. A.; Goebel, A.; Chen, S.; Mair, A.; Pan, J. W. Experimental realization of one-way quantum computing with two-photon four-qubit cluster states. Phys. Rev. Lett. 2007, 99, 120503.

(20) Lu, C.-Y.; Zhou, X.-Q.; Gühne, O.; Gao, W.-B.; Zhang, J.; Yuan, Z.-S.; Goebel, A.; Yang, T.; Pan, J.-W. Experimental entanglement of six photons in graph states. Nat. Phys. 2007, 3, 91-95.

(21) Kwiat, P. G.; Mattle, K.; Weinfurter, H.; Zeilinger, A.; Sergienko, A. V.; Shih, Y. New high-intensity source of polarization-entangled photon pairs. Phys. Rev. Lett. 1995, 75, 4337. 
(22) Zhong, H.-S.; Li, Y.; Li, W.; Peng, L.-C.; Su, Z.-E.; Hu, Y.; He, Y.-M.; Ding, X.; Zhang, W.; Li, H., et al. 12-Photon Entanglement and Scalable Scattershot Boson Sampling with Optimal Entangled-Photon Pairs from Parametric Down-Conversion. Phys. Rev. Lett 2018, 121, 250505.

(23) Wang, X.-L.; Luo, Y.-H.; Huang, H.-L.; Chen, M.-C.; Su, Z.-E.; Liu, C.; Chen, C.; Li, W.; Fang, Y.-Q.; Jiang, X.; Zhang, J.; Li, L.; Liu, N.-L.; Lu, C.-Y.; Pan, J.-W. 18-Qubit Entanglement with Six Photons' Three Degrees of Freedom. Phys. Rev. Lett. 2018, 120, 260502.

(24) Aharonovich, I.; Englund, D.; Toth, M. Solid-state single-photon emitters. Nat. Photonics 2016, 10, 631-641.

(25) Senellart, P.; Solomon, G.; White, A. High-performance semiconductor quantum-dot single-photon sources. Nat. Nanotechnol. 2017, 12, 1026-1039.

(26) Wang, H.; Hu, H.; Chung, T.-H.; Qin, J.; Yang, X.; Li, J.-P.; Liu, R.-Z.; Zhong, H.S.; He, Y.-M.; Ding, X. On-demand semiconductor source of entangled photons which simultaneously has high fidelity, efficiency, and indistinguishability. Phys. Rev. Lett. 2019, 122, 113602.

(27) Liu, J.; Su, R.; Wei, Y.; Yao, B.; da Silva, S. F. C.; Yu, Y.; Iles-Smith, J.; Srinivasan, K.; Rastelli, A.; Li, J. A solid-state source of strongly entangled photon pairs with high brightness and indistinguishability. Nat. Nanotechnol. 2019, 14, 586-593.

(28) Lindner, N. H.; Rudolph, T. Proposal for Pulsed On-Demand Sources of Photonic Cluster State Strings. Phys. Rev. Lett. 2009, 103, 113602.

(29) Economou, S. E.; Lindner, N.; Rudolph, T. Optically generated 2-dimensional photonic cluster state from coupled quantum dots. Phys. Rev. Lett. 2010, 105, 093601. 
(30) Pichler, H.; Choi, S.; Zoller, P.; Lukin, M. D. Universal photonic quantum computation via time-delayed feedback. Proc. Natl. Acad. Sci. U. S. A. 2017, 114, 11362-11367.

(31) Schwartz, I.; Cogan, D.; Schmidgall, E. R.; Don, Y.; Gantz, L.; Kenneth, O.; Lindner, N. H.; Gershoni, D. Deterministic generation of a cluster state of entangled photons. Science 2016, 354, 434-437.

(32) Wang, H.; Qin, J.; Ding, X.; Chen, M.-C.; Chen, S.; You, X.; He, Y.-M.; Jiang, X.; You, L.; Wang, Z.; Schneider, C.; Renema, J. J.; Höfling, S.; Lu, C.-Y.; Pan, J.W. Boson Sampling with 20 Input Photons and a 60-Mode Interferometer in a $10^{14}$ Dimensional Hilbert Space. Phys. Rev. Lett. 2019, 123, 250503.

(33) Wang, H.; Duan, Z. C.; Li, Y. H.; Chen, S.; Li, J. P.; He, Y. M.; Chen, M. C.; He, Y.; Ding, X.; Peng, C.-Z.; Schneider, C.; Kamp, M.; Höfling, S.; Lu, C.-Y.; Pan, J.W. Near-Transform-Limited Single Photons from an Efficient Solid-State Quantum Emitter. Phys. Rev. Lett. 2016, 116, 213601.

(34) He, Y.-M.; He, Y.; Wei, Y.-J.; Wu, D.; Atatüre, M.; Schneider, C.; Höfling, S.; Kamp, M.; Lu, C.-Y.; Pan, J.-W. On-demand semiconductor single-photon source with near-unity indistinguishability. Nat. Nanotechnol. 2013, 8, 213-217.

(35) Ding, X.; He, Y.; Duan, Z.-C.; Gregersen, N.; Chen, M.-C.; Unsleber, S.; Maier, S.; Schneider, C.; Kamp, M.; Höfling, S. On-demand single photons with high extraction efficiency and near-unity indistinguishability from a resonantly driven quantum dot in a micropillar. Phys. Rev. Lett. 2016, 116, 020401.

(36) Weber, J. H.; Kambs, B.; Kettler, J.; Kern, S.; Maisch, J.; Vural, H.; Jetter, M.; Portalupi, S. L.; Becher, C.; Michler, P. Two-photon interference in the telecom C-band after frequency conversion of photons from remote quantum emitters. Nat. Nanotechnol. 2019, 14, 23-26. 
(37) Deng, Y.-H.; Wang, H.; Ding, X.; Duan, Z.-C.; Qin, J.; Chen, M.-C.; He, Y.; He, Y.-M.; Li, J.-P.; Li, Y.-H., et al. Quantum Interference between Light Sources Separated by 150 Million Kilometers. Phys. Rev. Lett. 2019, 123, 080401.

(38) Hong, C.-K.; Ou, Z.-Y.; Mandel, L. Measurement of subpicosecond time intervals between two photons by interference. Phys. Rev. Lett. 1987, 59, 2044.

(39) Gisin, N.; Bechmann-Pasquinucci, H. Bell inequality, Bell states and maximally entangled states for n qubits. Phys. Lett. A 1998, 246, 1-6.

(40) Dür, W.; Briegel, H.-J. Stability of macroscopic entanglement under decoherence. Phys. Rev. Lett. 2004, 92, 180403.

(41) Sackett, C. A.; Kielpinski, D.; King, B. E.; Langer, C.; Meyer, V.; Myatt, C. J.; Rowe, M.; Turchette, Q. A.; Itano, W. M.; Wineland, D. J.; Monroe, C. Experimental entanglement of four particles. Nature 2000, 404, 256-259.

(42) Gühne, O.; Tóth, G. Entanglement detection. Phys. Rep. 2009, 474, 1-75.

(43) Wang, X.-L.; Chen, L.-K.; Li, W.; Huang, H.-L.; Liu, C.; Chen, C.; Luo, Y.-H.; Su, Z.E.; Wu, D.; Li, Z.-D., et al. Experimental Ten-Photon Entanglement. Phys. Rev. Lett 2016, 117, 210502.

(44) Pan, J.-W.; Daniell, M.; Gasparoni, S.; Weihs, G.; Zeilinger, A. Experimental demonstration of four-photon entanglement and high-fidelity teleportation. Phys. Rev. Lett. 2001, 86, 4435 .

(45) Kuhlmann, A. V.; Houel, J.; Ludwig, A.; Greuter, L.; Reuter, D.; Wieck, A. D.; Poggio, M.; Warburton, R. J. Charge noise and spin noise in a semiconductor quantum device. Nat. Phys. 2013, 9, 570-575.

(46) Pilnyak, Y.; Aharon, N.; Istrati, D.; Megidish, E.; Retzker, A.; Eisenberg, H. S. Simple 
source for large linear cluster photonic states. Phys. Rev. A: At., Mol., Opt. Phys. 2017, 95, 022304.

(47) Unsleber, S.; He, Y.-M.; Gerhardt, S.; Maier, S.; Lu, C.-Y.; Pan,; Gregersen, N.; Kamp, M.; Schneider, C. P.; Höfling, S. Highly indistinguishable on-demand resonance fluorescence photons from a deterministic quantum dot micropillar device with $74 \%$ extraction efficiency. Opt. Express 2016, 24, 8539-8546.

(48) Kolatschek, S.; Hepp, S.; Sartison, M.; Jetter, M.; Michler, P.; Portalupi, S. L. Deterministic fabrication of circular Bragg gratings coupled to single quantum emitters via the combination of in-situ optical lithography and electron-beam lithography. J. Appl. Phys. 2019, 125, 045701.

(49) Li, F.; Li, Y.; Cai, Y.; Li, P.; Tang, H.; Zhang, Y. Tunable Open-Access Microcavities for Solid-State Quantum Photonics and Polaritonics. Adv. Quantum Technol. 2019, 2, 1900060.

(50) Reindl, M.; Weber, J. H.; Huber, D.; Schimpf, C.; Covre da Silva, S. F.; Portalupi, S. L.; Trotta, R.; Michler, P.; Rastelli, A. Highly indistinguishable single photons from incoherently excited quantum dots. Phys. Rev. B: Condens. Matter Mater. Phys. 2019, 100,155420 .

(51) He, Y.-M.; Wang, H.; Wang, C.; Chen, M. C.; Ding, X.; Qin, J.; Duan, Z. C.; Chen, S.; Li, J. P.; Liu, R.-Z.; Schneider, C.; Atatüre, M.; Höfling, S.; Lu, C.-Y.; Pan, J.-W. Coherently driving a single quantum two-level system with dichromatic laser pulses. Nat. Phys. 2019, 15, 941-946.

(52) Wang, H.; He, Y.-M.; Chung, T.-H.; Hu, H.; Yu, Y.; Chen, S.; Ding, X.; Chen, M.-C.; Qin, J.; Yang, X. Towards optimal single-photon sources from polarized microcavities. Nat. Photonics 2019, 13, 770-775. 
(53) Huber, T.; Davanco, M.; Müller, M.; Shuai, Y.; Gazzano, O.; Solomon, G. S. Filter-free single-photon quantum dot resonance fluorescence in an integrated cavity-waveguide device. Optica 2020, 7, 380-385.

(54) Chang, D. E.; Vuletić, V.; Lukin, M. D. Quantum nonlinear optics — photon by photon. Nat. Photonics 2014, 8, 685-694.

(55) Kaneda, F.; Kwiat, P. G. High-efficiency single-photon generation via large-scale active time multiplexing. Sci. Adv. 2019, 5, eaaw8586.

(56) Joshi, C.; Farsi, A.; Clemmen, S.; Ramelow, S.; Gaeta, A. L. Frequency multiplexing for quasi-deterministic heralded single-photon sources. Nat. Commun. 2018, 9, 847.

(57) Bell, B. A.; Herrera-Martí, D. A.; Tame, M. S.; Markham, D.; Wadsworth, W. J.; Rarity, J. G. Experimental demonstration of a graph state quantum error-correction code. Nat. Commun. 2014, 5, 3658.

(58) Zhang, Q.; Bao, X.-H.; Lu, C.-Y.; Zhou, X.-Q.; Yang, T.; Rudolph, T.; Pan, J.-W. Demonstration of a scheme for the generation of "event-ready" entangled photon pairs from a single-photon source. Phys. Rev. A: At., Mol., Opt. Phys. 2008, r7, 062316.

(59) Erhard, M.; Krenn, M.; Zeilinger, A. Advances in High Dimensional Quantum Entanglement. arXiv:1911.10006 2019,

(60) Flamini, F.; Spagnolo, N.; Sciarrino, F. Photonic quantum information processing: a review. Rep. Prog. Phys. 2018, 82, 016001.

(61) Istrati, D.; Pilnyak, Y.; Loredo, J.; Antón, C.; Somaschi, N.; Hilaire, P.; Ollivier, H.; Cohen, L.; Vidro, L.; Millet, C., et al. Sequential generation of linear cluster states from a single photon emitter. arXiv:1912.04375 2019, 
Graphical TOC Entry

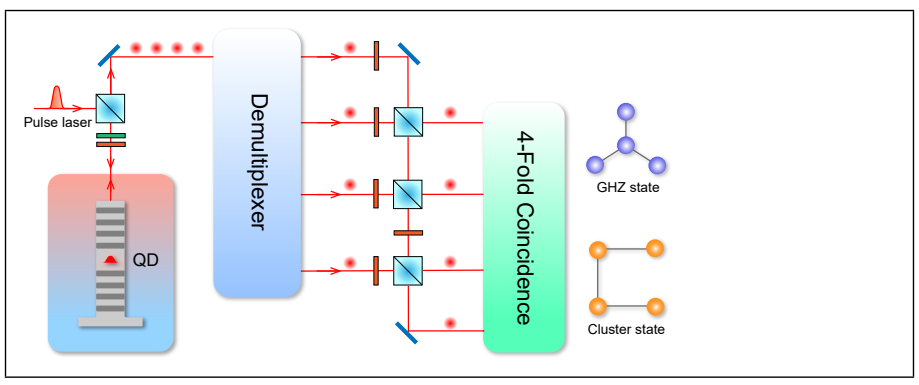

